\title{
Whole-Body Motion Planning for Pivoting Based Manipulation by Humanoids
}

\author{
Eiichi Yoshida, Mathieu Poirier, Jean-Paul Laumond, Oussama Kanoun, Florent Lamiraux, \\ Rachid Alami and Kazuhito Yokoi
}

\begin{abstract}
This paper emphasizes on the capacity of a humanoid robot to perform tasks that are difficult for other types of robots. It deals with manipulation of bulky objects. Such tasks require complicated manipulations involving the whole-body and fine coordination between legs, arms and torso motions. We introduce here a whole-body motion planner that allows a humanoid robot to autonomously plan a pivoting strategy that accounts for the various constraints: collision avoidance, legs-arms coordination and stability control. Based on a previous result by the authors [1] proving the small-time controllability of a pivoting system, the planner is proven to inherit from the probabilistic completeness of the samplingbased motion planning method it is built on. The geometric and kinematic capacity of the proposed planner is mainly demonstrated through simulations and experiments.
\end{abstract}

\section{INTRODUCTION}

Thanks to recent progress in their hardware and control, humanoid robots are expected to execute sophisticated tasks to assist or substitute humans. One of the important applications is dexterous manipulation of various objects, especially for bulky or heavy objects through whole-body motion. In this research we investigate pivoting based manipulation of a large object using both arms as shown in Fig. 1. This method has several advantages such as precise positioning, stability and adaptability over other methods like pushing or lifting [2], [3]. For those reasons, pivoting based manipulation can potentially widen the capacity of manipulation of humanoid robots.

This paper deals with motion planning in such a context. The inputs of the problem are both a starting and a goal configuration of the robot facing the box to be moved. The output is a sequence of box manipulations that guarantees:

- collision avoidance with the fixed obstacles

- feasibility and stability of the robot whole-body motions

- naturalness of the solution.

The solution we propose is derived from two geometric properties.

The first one establishes that any collision-free path for the free-sliding box may be approximated by a sequence of collision-free pivoting motions. Such a property has been

This work has been done as a joint research in AIST/IS-CNRS/ST2I Joint Japanese-French Robotics Laboratory (JRL).

Eiichi Yoshida and Kazuhito Yokoi are with JRL, Research Institute of Intelligent Systems, National Institute of Advanced Industrial Science and Technology (AIST), 1-1-1 Umezono, Tsukuba, Ibaraki 305-8568 Japan. \{e.yoshida, kazuhito.yokoi\} daist.go.jp

Mathieu Poirier, Jean-Paul Laumond, Oussama Kanoun, Florent Lamiraux and Rachid Alami are with JRL, LAAS-CNRS, University of Toulouse, 7 avenue du Colonel Roche 31077 Toulouse, France. \{mpoirier, jpl, okanoun, florent, rachid\} dlaas.fr

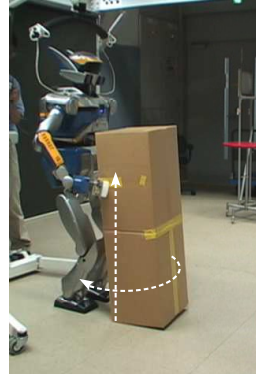

(a) Start rotating

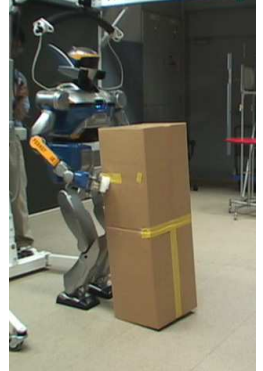

(b) Finish rotating

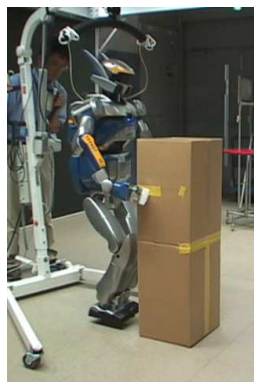

(c) Stepping
Fig. 1. Whole-body pivoting based manipulation. The humanoid robot inclines the object (a) and rotates it around vertical axis (b) using the support on a vertex. The robot makes a step to advance itself after several pivoting operations (c).

recently proven by the authors in [1]. As a consequence the motion planning algorithm we propose consider a two-stage approach: a first collision-free path is computed, and then it is iteratively approximated by a sequence of pivoting motions.

The second geometric property we are considering deals with the naturalness of the targeted solution. We want the robot to walk either forward or backward and to avoid sideways steps. Even if such steps are theoretically possible, they are not natural and even very difficult to perform by a humanoid robot when it manipulates bulky objects. When walking forward or backward the robot direction remains tangent to the path it follows as a wheeled mobile robot does. Such constraints are known as nonholonomic ones. They have been recently proven as accounting for the natural human locomotion [4]. Our motion planning point of view then benefits from well experienced approaches in nonholonomic motion planning [5], [6], [7]. Among them we have chosen the probabilistic sampling approach with a steering method computing Reeds and Shepp curves [8], composed of arc of a circle and straight line segments. Reeds and Shepp curves possess a geometric property accounting for small-time controllability, a critical property for the planning method completeness.

Thanks to these two properties it has been possible to devise a well-grounded motion planner we present in this paper. Section II deals with the computation of a collisionfree path without taking into account the pivoting constraints. It recalls a classical method for nonholonomic motion planning for a car-like robot. Then Section III emphasizes on pivoting sequence generation from the collision-free path generated by the planner introduced in the previous section. The whole-body motion generator to perform the pivoting 
tasks is devised based on a generalized inverse kinematics operator which is introduced in Section IV. Section V shows simulation and experimental results to validate the geometric and kinematic planning capacity of the proposed method, before concluding the paper.

\section{Collision-Free Motion Planning}

The first stage of the algorithm is dedicated to the computation of a collision-free nonholonomic path. For this stage we make use of a standard technique [5], [6], [7] we summarize here.

The robot together with the box is modeled as a rigid body whose geometry is their bounding volume. Such a rigid body is modeled as a car-like robot that is submitted to two constraints: its wheels should roll and not slide (this is the nonholonomic constraint), and the curvature of an admissible path at any point should be greater than some fixed threshold. The shortest length paths for such a system are the socalled Reeds and Shepp curves [8]. Reeds and Shepp curves are made of a sequence of arcs of a circle with minimum radius and straight line segments. The interest of Reeds and Shepp curves is the following: their length tends to zero as soon as the goal configuration $\left(x_{g}, y_{g}, \theta_{g}\right)$ tends to the start configuration $\left(x_{s}, y_{s}, \theta_{s}\right)$.

To compute a collision-free nonholonomic path we use a probabilistic roadmap method (PRM) that consists is building a graph whose nodes are collision-free configurations. Two nodes in the graph are linked by an edge if and only if the Reeds and Shepp path between both corresponding configurations is collision-free. The graph is built incrementally by shooting configurations at random. Configurations are included in the graph as soon as they are collision-free (from a practical point of view we use a more sophisticated and efficient variant described in [9]). This construction of the graph is called the learning phase. Once the graph is built, then the query phase consists in first adding both starting and goal configurations of the given problem to the roadmap, and then search the graph for a path.

In that way, the motion planner eventually finds collisionfree paths as connected components of elementary motions of the steering method. However, they are often redundant since the sampling-based method samples configuration randomly. For manipulation to be more efficient, redundant components are removed or shortened. The path optimizer plays this role. It searches shorter paths by connecting configuration pairs randomly sampled on the initial path. In this study we employ the "adaptive shortcut" path optimization algorithm proposed by [12].

Figure 2 shows an example of optimized path. The manipulated object is placed near the wall and supposed to be displaced on the other side of an obstacle. We modeled as a bounding box including the humanoid and the object with some tolerance necessary for the pivoting based manipulation. As can be seen, the backward motion of Reeds and Shepp curve is utilized appropriately to move the object away from the wall. Then the path switches to forward motion to reach the goal by avoiding the obstacle.

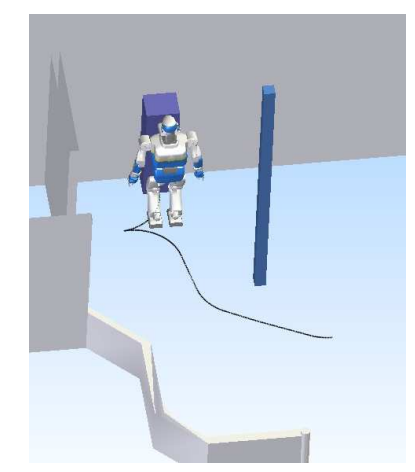

(a) Initial state

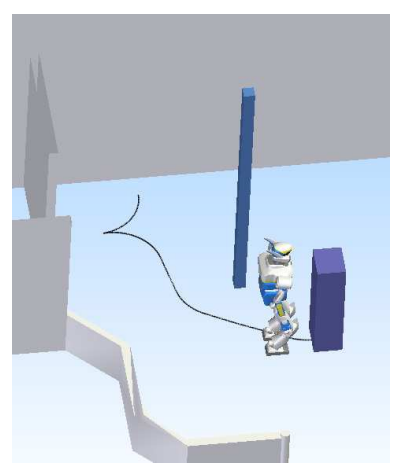

(b) Goal State
Fig. 2. Optimized collision-free path for a manipulated box object and the humanoid robot using Reeds and Shepp curves. The path allows the humanoid to move the object away from the wall starting from the initial state (a) by taking advantage of backward motion. Then the path switches to forward motion to avoid obstacle and to move the object to the goal (b).

\section{Pivoting Sequence Generation}

In this section we present how to convert the collisionfree path computed at the first stage into a sequence of collision-free pivoting sequences. The path to be converted is a sequence of Reeds and Shepp path, i.e. a sequence of straight line segments and arcs of a circle. The pivoting sequence generation is then based on two elementary operators: pivoting along a straight line segment and pivoting along an arc of a circle.

Let us first analyze the effective strategy used by the robot to perform an elementary pivoting motion. The robot starts inclining the box to realize a single contact point between the box and the floor. The contact point is a corner of the box. Then the robot performs a vertical rotation of the box centered at that corner. Then it sets the object horizontally along the box edge. Such an edge is said to be the supporting edge. Therefore we model the problem of 3D box pivoting as the problem of pivoting a 2D segment around its endpoints (see Fig. 3). Such a modeling does not reduce the scope of the general problem ${ }^{1}$.

The computation of the pivoting sequence along a straight line segment is illustrated in Fig. 3. Let $l$ be the length of the segment corresponding to the "supporting edge" and $L$ the length of the straight line segment of the path to follow. Considering the constraint of the reachable area of robot arms, we introduce an angle $\beta$ such that the robot is able to perform an elementary pivoting motion of total angle $2 \beta$. After initializing the process by a pivoting of angle $\beta$, we then apply $n$ times the elementary pivoting motion of angle $2 \beta, n$ being defined as the greater integer verifying $L>n l \sin \beta$. Then for the last part an adjustment pivoting motion is added to reach the final goal.

The same principle applies to the arcs of a circle (see Fig. 4). Let $R$ and $\theta$ denote the radius and the angle of the arc. We apply a regular sequence shown in Fig. 4 with a symmetrical motion such that the center of the line segment

\footnotetext{
${ }^{1}$ Notice that we do consider the cases where the robot would overcome small obstacles on the floor by pivoting the box above the obstacles.
} 


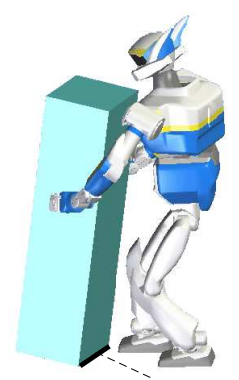

Supporting edge

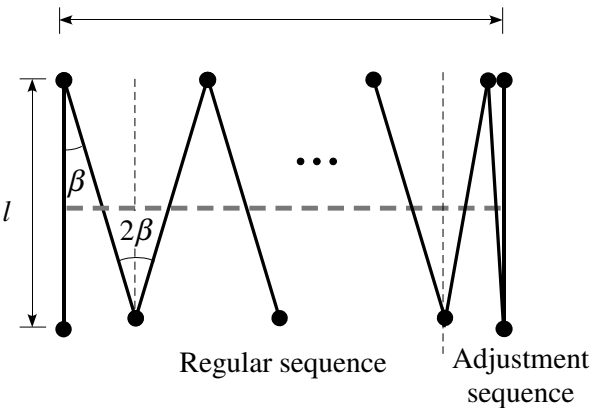

sequence
Fig. 3. Transforming a straight line segment path into a pivoting sequence. The pivoting sequence is planned using rotation of the endpoints of the supporting edge (left). During the regular sequence, rotations of same angles are repeated before adjustment sequence that positions the line segment at the endpoint.

comes on the arc with perpendicular orientation, after two times $\beta$ rotation at the left corner and one $-2 \gamma$ rotation at the right corner. The angle $\alpha$ and $\gamma$ can be computed from $l, R$, and $\beta$ as:

$$
\begin{aligned}
\alpha & =\arctan \left(\frac{l \sin \beta}{R-\frac{l}{2}+l \cos \beta}\right), \\
\gamma & =\beta-\alpha .
\end{aligned}
$$

In the regular sequence, elementary motions are repeated $m$ times alternatively on the each edge, while $\theta>m \alpha$. Then the adjustment sequence is applied in a similar manner to the computation for the straight line path.

We introduced the angle $\beta$ as half the maximum angle the robot may realize by a single pivoting. We should notice that this angle may be tuned for obstacle avoidance purpose. Indeed the first stage of the algorithm provides a collisionfree path that guarantees collision-freeness for the sliding supporting edge. Moving by pivoting along the planned path introduces some gap (see Fig. 5) with respect to the volume swept by the supporting edge when sliding along the path. More the rotation angle decreases, more the final swept

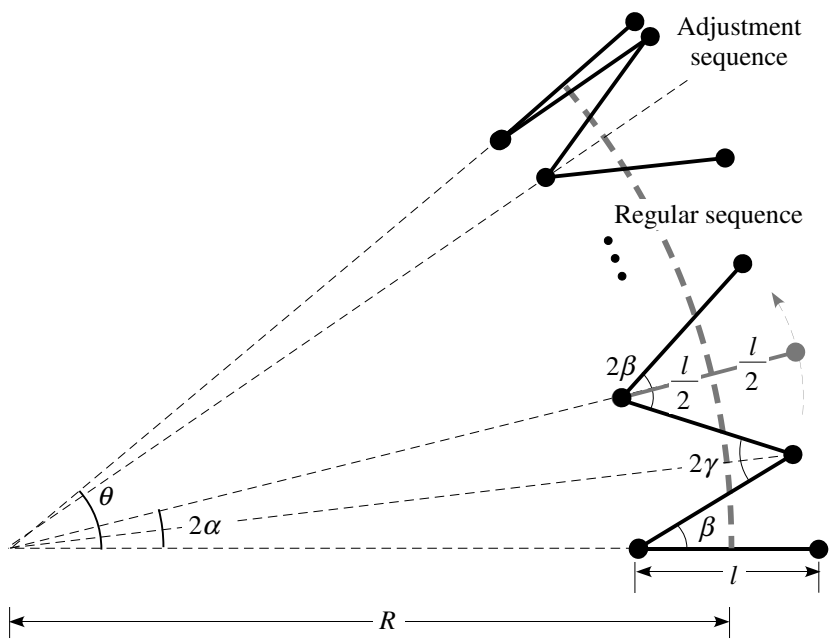

Fig. 4. Transforming an arc of circle path into a pivoting sequence. The regular sequence is composed of symmetric rotations. The center of pivoting line segment arrives at the end point of the arc with the perpendicular orientation after adjustment sequence.

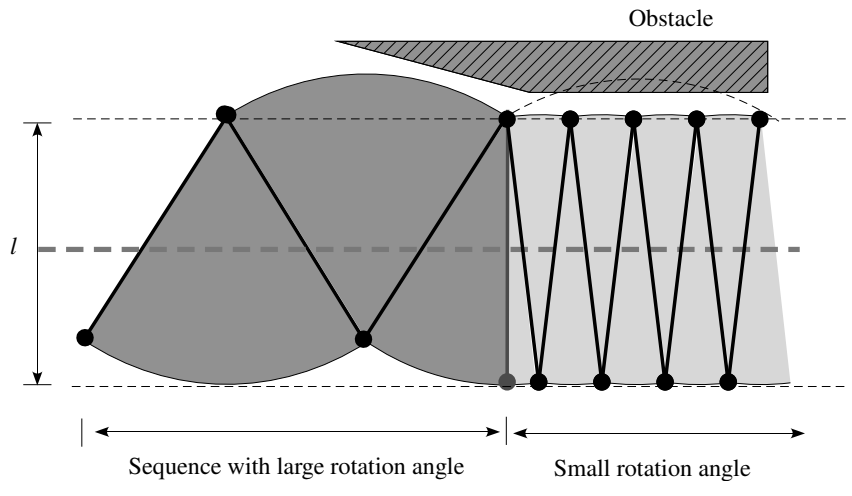

Fig. 5. The swept volume of pivoting. It converges to the swept volume of the supporting edge along the straight line segment by reducing the rotation angles. Therefore, the original collision-free Reeds and Shepp path can be converted into a collision-free pivoting sequence.

volume converges to the initial one. This property accounts for the small-time controllability of the pivoting system we consider [1]. The 3D collision detection can be done by estimating the swept volume of the box attached to the supporting edge during the rotational motion. The inclination for pivoting can be taken into account by using a bounding volume including the inclining motion of the box.

As a consequence the two step strategy we have developed inherits from the probabilistic completeness of the motion planner used at the first stage. The approximation scheme by on pivoting sequence generation does not introduce any incompleteness.

\section{Whole-Body Humanoid Motion Generation}

The generated pivoting sequence should be realized by the humanoid robot by using its two arms. The humanoid motion should be generated in such a way that constraints like dynamic balancing and arm manipulation motion are satisfied at the same time. Moreover, stepping motion should be added in order to continue the manipulation when necessary.

For this purpose we adopt a general framework of wholebody motion generation [13] including dynamic stepping motion. Based on a generalized inverse kinematic (IK) method (e.g., [14], [15]), such tasks as center of mass (CoM) position, stepping and hand motion are treated with priorities. Since all the joints are involved to make those complicated combined motions, we can expect better performance in the sense of reachable space than a functional decomposition utilized in [3].

The method is illustrated in Fig. 6. In the case of pivoting, the solver is given trajectories of hands, feet or CoM as well as their position and orientation constraints as prioritized tasks. The generalized IK solver computes the whole-body motion as joint angle trajectories.

In this framework there is a motion manager that updates the configuration of the robot and objects during the computation. Hand trajectories for pivoting are supplied to the manager to organize how the next motions are generated. The motion generation process is branched into two cases depending on the distance between robot and the object. Note 


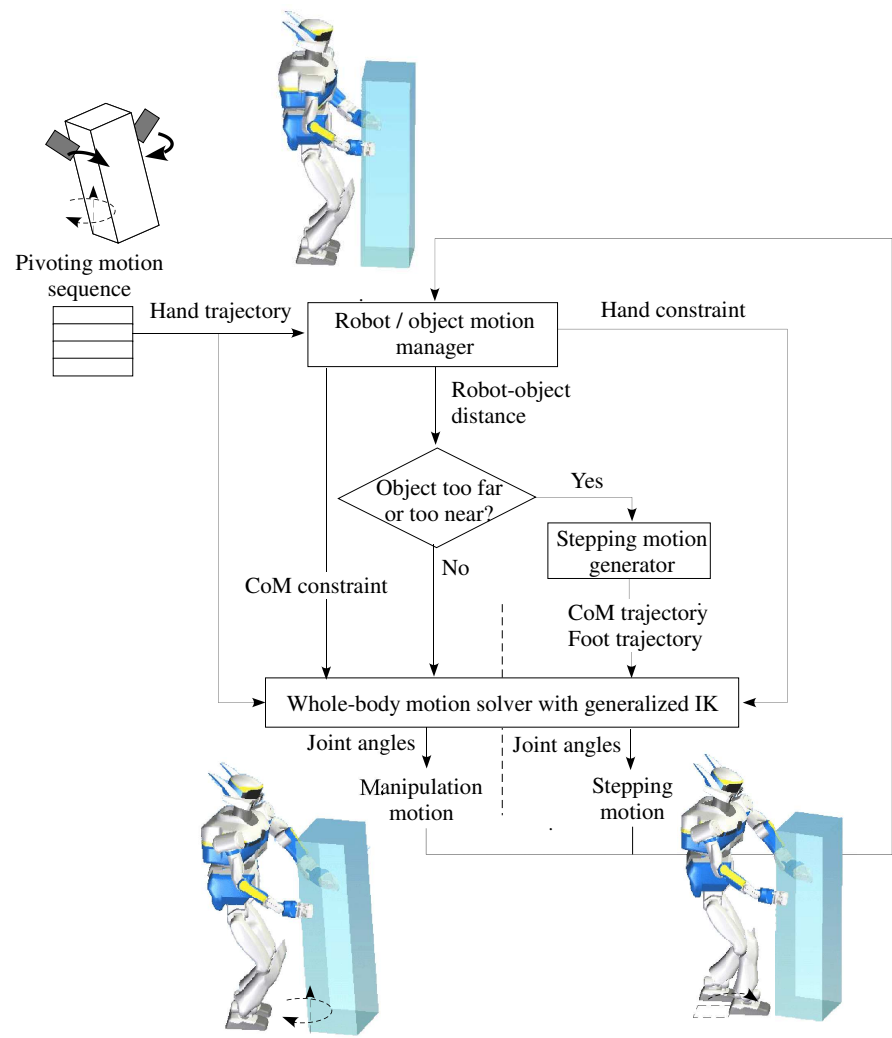

Fig. 6. Usage of generalized inverse kinematics utilized for whole-body motion for pivoting based manipulation.

that we use the same framework of generalized IK solver is utilized for both cases.

If the object stays within the range where the manipulation is feasible, the generalized IK solver takes the hand trajectory and the CoM constraint as inputs. It then outputs a whole-body motion that allows the humanoid to perform the manipulation keeping the balance as shown in the left side of Fig. 6.

The robot takes forward or backward stepping motions when the object is located too far or too near. The hand positions are kept constant during the stepping motion, which is given to the generalized IK solver as constraints. The dynamically stable stepping motion is generated as trajectories of CoM and foot using Zero Moment point (ZMP) based preview controller proposed in [16]. The generalized IK solver takes those trajectories and constraints as input to obtain a stepping motion keeping the hand positions (right side of Fig. 6). This process is applied to each pivoting operation in the sequence.

\section{SimUlations AND EXPERIMENTS}

The proposed planning scheme is applied to the pivoting based manipulation by humanoid robot HRP-2 [17] by using the motion planning software kit KineoWorks ${ }^{\mathrm{TM}}$ [18] and the dynamic robot simulator and controller OpenHRP [19]. We have been developing a common software framework "Humanoid Path Planner" (Fig. 7) that implements basic functionalities of motion planning for systems like humanoids or digital actors [20]. Its object-oriented architecture allows the users to define our planner of the whole-body pivoting motion as an inherited class of a general robot motion planner. The planner takes care of interaction with basic functions such as roadmap builder, path optimizer and collision checker. We also implemented the steering methods specific to the pivoting planning problem.

\section{A. Planning Results}

The same environment as in Fig. 2 is utilized for wholebody motion planning for pivoting of a box object. The radius is fixed as $0.5 \mathrm{~m}$ for Reeds and Shepp curves and the width, depth and height of the box is $0.35 \mathrm{~m}, 0.3 \mathrm{~m}$ and $1.2 \mathrm{~m}$. The robot holds the object by point contact on the side faces at the point at $0.8 \mathrm{~m}$ and $0.05 \mathrm{~m}$ from the bottom and front face respectively. The rotation angle $\beta$ is set to $15^{\circ}$.

Fig. 8 shows some snapshots of planned result of motion planning for pivoting based manipulation. We can observe the whole-body motion where overall joints participate in pivoting or stepping. The humanoid robot makes a backward motion to move the box object away from the wall along an arc of circle. As the upper snapshots shows, the robot moves the object backward by combining motions of pivoting and stepping back. The lower snapshot shows forward pivoting along a straight line segment.

The computation time is in the order of several hundreds of milliseconds for each of Reeds and Shepp curve and pivoting sequence generation using a PC of Pentium M $2.1 \mathrm{GHz}$. The computation of whole-body motion takes about half of the manipulation task duration time including calculation of generalized inverse kinematics is for the control command (joint angles and reference ZMP) of the hardware robot at the sampling time of $10 \mathrm{~ms}$.

\section{B. Experimental Results}

We here show an experimental result of planned wholebody pivoting motion. The generated motions are sent to the onboard computer that takes charge of real-time motion control for the humanoid. The controller and stabilizer implemented on OpenHRP [19] finally execute the planned motions on the humanoid hardware.

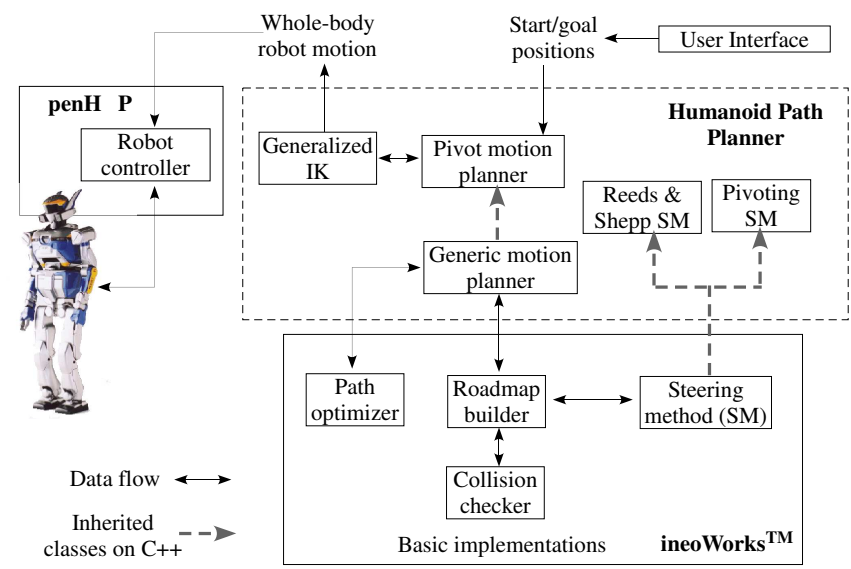

Fig. 7. Architecture of Humanoid Path Planner framework that facilitates implementation of robotic motion planner according to specific problems. 


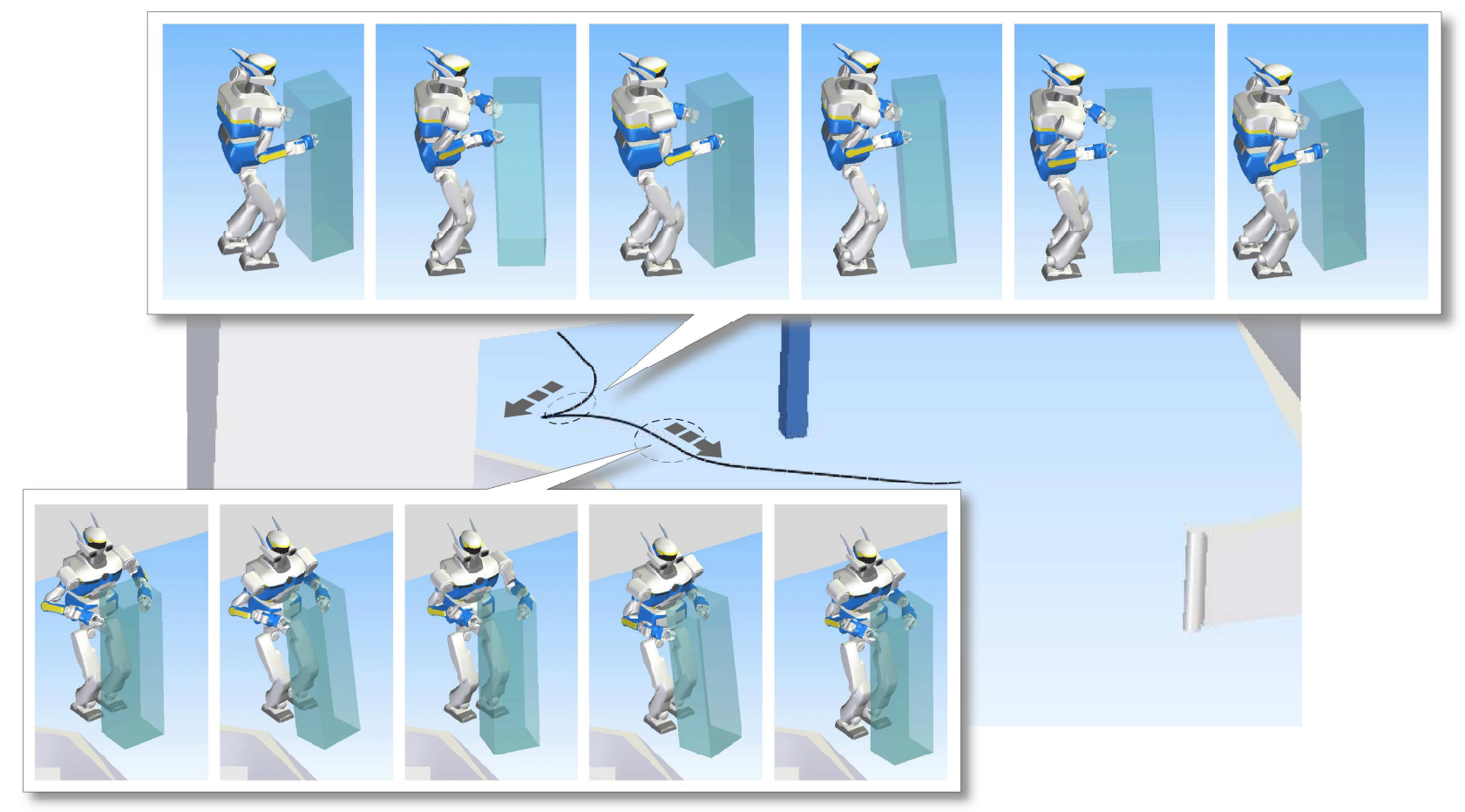

Fig. 8. Snapshots of the planned result. The manipulated object is drawn transparently to show the robot motion. The Reeds and Shepp path allows the robot to use backward motions to move the object close to the wall. The snapshots show how the humanoid robot transports the object through combination of pivoting and stepping motions along the planned optimized Reeds and Shepp path.

We have verified the generated whole-body motion for pivoting based manipulation using a straight line segment path. The same size of the box as the planning is used with the weight of $1.5 \mathrm{~kg}$ and the length of the test path is $0.35 \mathrm{~m}$. In this example we reduced $\beta$ to $5^{\circ}$ for security. The incline angle and the maximum velocity at the hand are $5^{\circ}$ and 0.15 $\mathrm{m} / \mathrm{s}$ respectively. The sequence in the experiment includes 12 pivoting operations and the total task time is 80 seconds.

As a result, the object has been successfully displaced to the goal position. Figures 9 and 10 show some pictures of a pivoting sequence and stepping motion in the experiment. In those pictures we can confirm that the whole-body motions generated by the proposed method have been smoothly performed by the hardware robot. In Fig 9, the robot displaced the box object by supporting it on its right corner. The stepping motion is performed in such a way that the relative hand positions and orientations with respect to the box are maintained in Fig 10.

The whole-body motion can be clearly observed in the movies of the experiment is available on (http://www.laas.fr/7 Eyoshida/icra2008/pivot/).

\section{CONCLUSIONS}

Moving large objects is one of the capabilities offered by human-size humanoid robots. In this paper we have seen it is possible to benefit from well-grounded robot algorithms and software to address robot autonomy in such a difficult context. Based on a formal analysis of the task to be performed, we have devised an integrated approach for manipulation task planning and control that accounts for a large class of constraints ranging from obstacle avoidance to whole-body motion control. The approach takes advantage of a controllability analysis previously published in [1] that reduces the problem of existence of a manipulation path to that of the existence of a path that ignores manipulation constraints. Then we have shown that the collision avoidance strategy may benefit from well-experienced techniques in nonholonomic motion planning, including probabilistic sampling approaches. As dynamic motion control is concerned we benefit from a generalized inverse kinematic approach that accounts for ZMP control [13], and also from the dynamic motion simulator OpenHRP [19].

This paper concentrates on the geometric and kinematic aspect of whole-body manipulation planning and dynamically stable robot motions for manipulation and stepping have been successfully generated. Physical aspects for manipulation of heavier objects will be addressed in the next developments. Further works also open questions of manipulation planning when grasping changes [21] or environment rearrangements [22] are required. Such questions start being to be addressed in humanoid robotics [23]. They are promising routes for the future applications putting humanoid robots at work.

\section{ACKNOWLEDGMENT}

We also thank to Dr. Neo Ee Sian and Mr. Hiroki Sanada of AIST for their precious help for the experiments. This research was partially supported by Japan Society for the Promotion of Science (JSPS) Grant-in-Aid for Scientific Research (B), 18300070, 2006. 


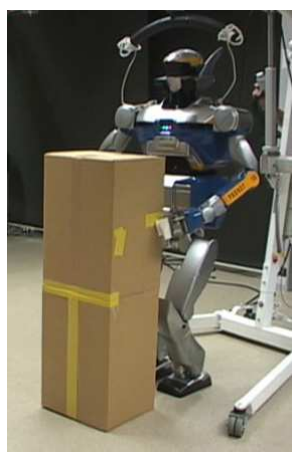

(a) Initial state

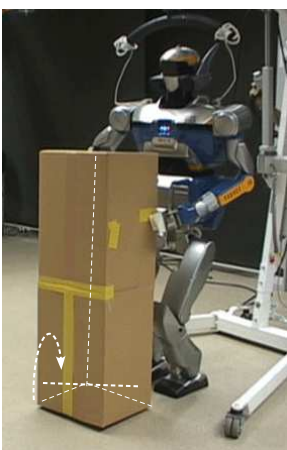

(b) Inclining

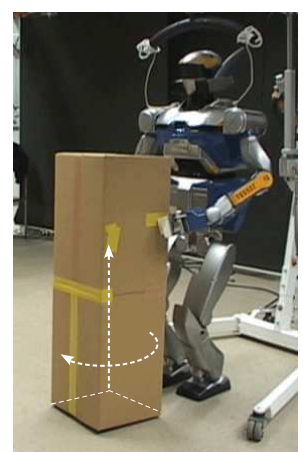

(c) Rotation

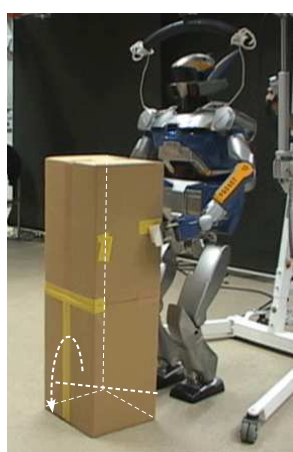

(d) Inclining back

Fig. 9. A sequence of three elementary rotation realized by whole-body motion in pivoting based manipulation in the experiment. The object is first inclined to support on one corner (a) and next rotated along the vertical axis (b) and finally inclined back to go back to the horizontal position.

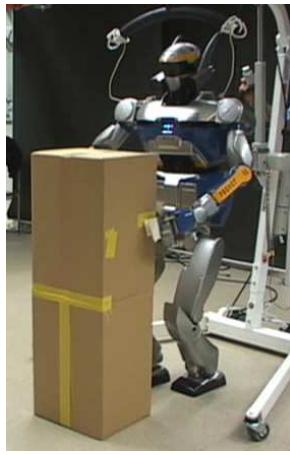

(a) Before stepping

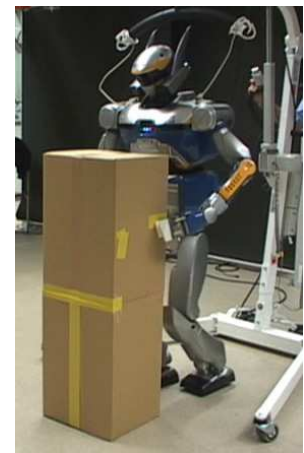

(b) Start stepping

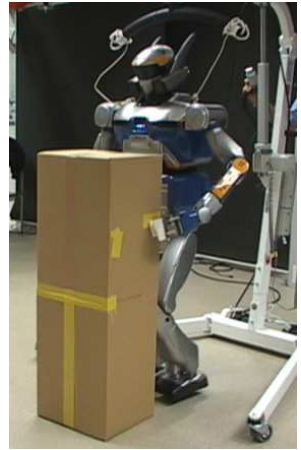

(c) Single support phase

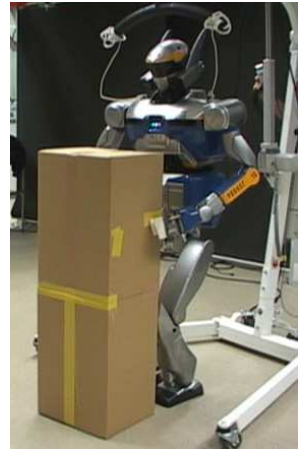

(d) End stepping

Fig. 10. Stepping motion between two pivoting operation in the experiment. The robot goes to single support phase (b). Dynamic stepping motion is generated (c) before going back to double support phase (d). The relative hand position and orientation to the box are maintained during stepping.

\section{REFERENCES}

[1] E.Yoshida, M. Poirier, J.-P. Laumond, R. Alami and K. Yokoi: "Pivoting Based Manipulation by Humanoids: a Controllability Analysis," Proc. IEEE/RSJ Int. Conf. on Intelligent Robots and Systems, 1130 1135, 2007.

[2] Y. Aiyama, M. Inaba, and H. Inoue: "Pivoting: A new method of graspless manipulation of object by robot fingers," Proc. IEEE/RSJ Int. Conf. on Intelligent Robots and Systems, 136 - 143, 1993.

[3] E. Yoshida, P. Blazevic, V. Hugel, K. Yokoi and K. Harada: "Pivoting a large object: whole-body manipulation by a humanoid robot," J. of Applied Bionics and Biomechanics, 3-3, 227-235, 2006.

[4] G. Arechavaleta, J-P. Laumond, H. Hicheur and A. Berthoz., "The nonholonomic nature of human locomotion: a modeling study," Proc. 2006 IEEE / RAS-EMBS Int. Conf. on Biomedical Robotics and Biomechatronics, 158 - 163, 2006.

[5] J.-P. Laumond (editor), "Robot Motion Planning and Control," Lectures Notes in Control and Information Sciences 229, Springer, 1998.

[6] H. Choset, K. Lynch, S. Hutchinson, G. Kantor, W. Burgard, L. Kavraki and S. Thrun, "Principles of Robot Motion: Theory, Algorithms, and Implementation," MIT Press, 2006.

[7] S. LaValle. "Planning Algorithms," Cambridge Univ. Press, 2006.

[8] J. A. Reeds and R. A. Shepp, "Optimal paths for a car that goes both forwards and backwards," Pacific Journal of Mathematics, 145-2, 367393, 1990.

[9] T. Simeon, J.-P. Laumond and C. Nissoux, "Visibility-based probabilistic roadmaps for motion planning," J. of Advanced Robotics, 14-6, 477-494, 2000.

[10] S. LaValle and J. Kuffner, "Rapidly-Exploring Random Trees: Progress and Prospects," Algorithmic and Computational Robotics: New Directions, 293-308, A K Peters, 2001.

[11] L. Kavraki, P. Svestka, J.-C. Latombe, and M. Overmars, "Probabilistic Roadmaps for Path Planning in High-Dimensional Configuration Spaces," IEEE Trans. on Robotics and Automation, 12-4, 566-580, 1996.
[12] D. Hsu. Randomized Single-query Motion Planning in Expansive Spaces. Ph.D. Thesis, Dept. of Computer Science, Stanford University, Stanford, CA, 2000.

[13] E. Yoshida, O. Kanoun, J.-P. Laumond and C. Esteves, "Task-driven Support Polygon Reshaping for Humanoids," Proc. IEEE-RAS Int. Conf. on Humanoid Robots, 208-213, 2006.

[14] Y. Nakamura, "Advanced Robotics: Redundancy and Optimization," Addison-Wesley Longman Publishing, Boston, 1991.

[15] B. Siciliano and J.-J. E. Slotine, "A general framework for managing multiple tasks in highly redundant robotic systems," Proc. IEEE Int. Conf. on Advanced Robotics, 1211-1216, 1991.

[16] S. Kajita, et al., F. Kanehiro, K. Kaneko, K. Fujiwara, K. Harada, K. Yokoi and H. Hirukawa, "Biped Walking Pattern Generation by using Preview Control of Zero-Moment Point," Proc. IEEE Int. Conf. on Robotics and Automation, 1620-1626, 2003.

[17] K. Kaneko, F. Kanehiro, S. Kajita, H. Hirukawa, T. Kawasaki, M. Hirata, K. Akachi and T. Isozumi, "The Humanoid Robot HRP-2", Proc. IEEE Int. Conf. on Robotics and Automation, 1083-1090, 2004.

[18] J-P. Laumond, "Kineo CAM: a success story of motion planning algorithms," IEEE Robotics \& Automation Magazine, 13-2, 90-93, 2006.

[19] F. Kanehiro, H. Hirukawa and S. Kajita, "OpenHRP: Open Architecture Humanoid Robotics Platform," Int. J. of Robotics Research, 23-2, 155-165, 2004.

[20] A. Mallet, F. Kanehiro, S. Fleury and M. Herrb, "Reusable Robotics Software Collection," Second Int. Workshop on Software Development and Integration in Robotics (SDIR), 2007.

[21] T. Simeon, J-P. Laumond, J. Cortes, A. Sahbani, "Manipulation planning with probabilistic roadmaps," Int. J. Robotics Research, 23, 7-8, 729-746, 2004

[22] M. Stilman and J. Kuffner, "Planning among movable obstacles with artificial constraints," Proc. 6th Int. Workshop on the Algorithmic Foundations of Robotics (WAFR'06), 1-20, 2006.

[23] M. Stilman, K. Nishiwaki, S. Kagami, and J. Kuffner, "Planning and executing navigation among movable obstacles," Proc. IEEE/RSJ Int. Conf. on Intelligent Robots and Systems, 820-826, 2006. 